\title{
Impact of Cotransplantation of Mesenchymal Stem Cells on Lung Function After Unrelated Allogeneic Hematopoietic Stem Cell Transplantation Following Non-Myeloablative Conditioning
}

\author{
Catherine Moermans, ${ }^{1,5}$ Chantal Lechanteur, ${ }^{2}$ Etienne Baudoux, ${ }^{2}$ Olivier Giet, ${ }^{2}$ Monique Henket, ${ }^{1}$ \\ Laurence Seidel, ${ }^{3}$ Marie Lejeune, ${ }^{4}$ Evelyne Willems, ${ }^{4}$ Frederic Baron, ${ }^{4}$ Renaud Louis, ${ }^{1}$ and Yves Beguin ${ }^{2,4}$
}

\begin{abstract}
Background. In the context of hematopoietic stem cell transplantation (HSCT), mesenchymal stem cells (MSC) have been used to promote engraftment and prevent graft-versus-host disease. However, in animal models, MSC were shown to cause pulmonary alterations after systemic administration. The impact of MSC infusion on lung function has not been studied in humans. The objective of the study was to investigate the impact of MSC co-infusion on lung function and airway inflammation as well as on the incidence of pulmonary infections and cytomegalovirus (CMV) reactivation after HSCT.

Methods. We have prospectively followed 30 patients who underwent unrelated HSCT with MSC co-infusion after non-myeloablative conditioning (NMA). Each patient underwent detailed lung function testing $\left(\mathrm{FEV}_{1}, \mathrm{FVC}, \mathrm{FEV}_{1} /\right.$ FVC, RV, TLC, DLCO, and KCO) and measurement of exhaled nitric oxide before HSCT and 3, 6, and 12 months posttransplant. The incidence of pulmonary infections and CMV reactivation were also monitored. This group was compared with another group of 28 patients who underwent the same type of transplantation but without MSC co-infusion. Results. Lung function tests did not show important modifications over time and did not differ between the MSC and control groups. There was a higher 1-year incidence of infection, particularly of fungal infections, in patients having received a MSC co-infusion. There was no difference between groups regarding the 1-year incidence of CMV reactivation. Conclusions. MSC co-infusion does not induce pulmonary deterioration 1 year after HSCT with NMA conditioning. MSC appear to be safe for the lung, but close monitoring of pulmonary infections remains essential.
\end{abstract}

Keywords: MSC, Stem cell transplantation, Lung function, Exhaled nitric oxide.

(Transplantation 2014;98: 348-353)

$\mathrm{M}$ esenchymal stem cells (MSC) are pluripotent cells derived from the bone marrow or other sources, which have stimulated a high level of enthusiasm in recent years for their potential therapeutic use.

The study was financially supported by TELEVIE (Grant F.N.R.S. 7.4.642.09.F) associated with the Fonds National de la Recherche Scientifique (FNRS) Belgium, PAI 6/35, and Fonds Léon Frédéricq.

The authors declare no conflicts of interest.

${ }^{1}$ Department of Pneumology-Allergology, CHU of Liege, 4000 Liege $\mathrm{I}^{3}$ Group, GIGA Research Center, University of Liege, Liege, Belgium.

${ }^{2}$ Laboratory of Cell and Gene Therapy, CHU of Liege, Liege, Belgium.

${ }^{3}$ Department of Statistics, University of Liege, Liege, Belgium.

${ }^{4}$ Department of Hematology, CHU of Liege, 4000 Liege I ${ }^{3}$ Group, GIGA Research Center, University of Liege, Liege, Belgium.

${ }^{5}$ Address correspondence to: Catherine Moermans, Ph.D., Department of Pneumology-Allergology, CHU of Liege, Avenue de l'hopital, 134000 Liege, Belgium.

E-mail: catherinemoermans@hotmail.com

C.M. participated in writing the article, performing the research, and analyzing the data, and was supported by Grant F.N.R.S. 7.4.642.09.F associated with the Fonds National de la Recherche Scientifique (FNRS) Belgium, PAI 6/35, and Fonds Léon Frédéricq. C.L. participated in procuring bone marrow from MSC donors, supervising the preparation of MSC products, and ensuring quality management of MSC production. E.B.
In the context of allogeneic hematopoietic stem cell transplantation (HSCT), MSC cotransplantation appears to be safe $(1,2)$. These cells could have a role in engraftment promotion (3) and graft-versus-host disease (GVHD) prevention (4) through their immunosuppressive activity. Because

participated in collecting bone marrow from MSC donors, preparing the MSC products, and ensuring quality management of MSC production. O.G. participated in preparing the MSC products and ensuring quality management of MSC production. M.H. participated in analyzing the data. L.S. participated in performing statistical analysis. M.L. participated in providing patient care and collecting clinical data. F.B. participated in providing patient care, collecting clinical data, and writing the article. E.W. participated in providing patient care, collecting clinical data, and writing the article. R.L. participated in writing the article. Y.B. participated in designing the study, providing patient care, and writing the article.

Supplemental digital content (SDC) is available for this article. Direct URL citations appear in the printed text, and links to the digital files are provided in the HTML text of this article on the journal's Web site (www.transplantjournal.com).

Received 6 November 2013. Revision requested 1 December 2013. Accepted 13 January 2014.

Copyright (C) 2014 by Lippincott Williams \& Wilkins

ISSN: 0041-1337/14/983-348

DOI: $10.1097 /$ TP.0000000000000068 
of their lack of major histocompatibility complex molecule expression, MSC are weakly immunogenic in humans, allowing administration to patients without HLA matching (5).

However, in some animal models, MSC infusion has been shown to cause pulmonary alterations. Indeed, after IV injection in mice, the cells are trapped within the pulmonary capillaries, thereby causing embolism (6). Moreover, mouse MSC could differentiate into tumor cells in lungs after systemic administration (7) and could promote tumor development (8). Another study in mice disclosed differentiation of MSC in the lung after irradiation, depending on the infusion timing (9): MSC differentiated into functional lung cells if injected at an early stage or into cells involved in fibrosis if injected once chronic inflammation and fibrosis had started. Furthermore, Salazar et al. showed evidence of a mitogenic potential of human and mouse MSC on lung fibroblasts in vitro (10). In contrast, other papers have shown that, in response to injury caused by endotoxin or bleomycin, MSC migrated to the lung, decreased tissue damage, and improved lung repair $(11,12)$. In addition, in rats with chronic obstructive lung disease, intra-tracheal MSC administration restored lung function (13). These observations may result from the ability of MSC to secrete paracrine growth factors and cytokines able to decrease inflammation. Moreover, the ability of MSC to differentiate into functional cells may be a key in promoting adequate lung repair. MSC are also suggested to be able to attenuate oxidative stress in inflammatory lung diseases induced by previous irradiation or by subclinical pathogen colonization in a context of immunosuppression (14).

The impact on the lung of MSC co-infusion after HSCT has not been studied so far in humans. In this study, we monitored the evolution of lung function, the value of exhaled nitric oxide (FeNO), and the occurrence of pulmonary infections and cytomegalovirus (CMV) reactivation in patients who underwent HSCT with MSC co-infusion. We also investigated the impact of MSC co-infusion on CMV reactivation and pulmonary infections in univariate and multivariate Cox models adjusted for competing risks.

It is widely accepted that performing pulmonary function tests (PFT) before and after transplantation is crucial to detect early signs of pulmonary complications. Lung function assessment before transplantation usually serves as baseline reference to evaluate changes after HSCT (15). We followed 30 patients who received HSCT for hematological malignancies from unrelated donors and a co-infusion of MSC after non-myeloablative conditioning (NMA). The results were compared with those of 28 patients who received the same type of transplant but without MSC. Pulmonary function parameters, including airway flow rates, lung volumes, and diffusing capacity (DLCO), as well as FeNO value, were measured before HSCT as well as 3, 6, and 12 months posttransplantation. FeNO is a noninvasive marker of airway inflammation used to monitor rejection after lung transplantation (16), but its utility has not been really assessed after HSCT.

\section{RESULTS}

At baseline, all patients displayed normal spirometric and lung volume values but a slight impairment of diffusing capacity. The median FeNO value was within the accepted normal range for both patient groups (17).

MSC co-infusion had no detrimental effect on lung function indices when expressed as predicted percentages (see Table S1, SDC, http://links.lww.com/TP/A951). When expressed as absolute value (data not shown), MSC co-infusion was even associated with a slight improvement in FEV1 (from $2.7 \pm 0.9 \mathrm{~L}$ at baseline to $2.9 \pm 0.9 \mathrm{~L}$ at 3 months, $P<0.05$ ) and FVC (from $3.6 \pm 1.1 \mathrm{~L}$ at baseline to $3.8 \pm 1.1$ at 6 months, $P<0.05)$ at some time points, which contrasted with the decrease in DLCO seen at 1 year in the group without MSC Coinfusion (from $6.3 \pm 1.8$ at baseline to $5.8 \pm 1.1$ at 1 year, $P<0.05$ ).

The other lung parameters (FEV1/FVC, KCO, TLC, RV, and FeNO) were not significantly changed after 3, 6, or 12 months in any of the patient groups and did not show differences between groups when expressed as predicted percentages or absolute values.

The 1-year cumulative incidence of pulmonary infection appeared higher in the MSC group compared to the control group $(P<0.01$; see Table 1 and Figure S1A, SDC, http://links.lww.com/TP/A951). Infection etiologies showed a trend for a higher rate of fungal infections in the MSC group ( 6 vs. $1 ; P=0.06$ ). In contrast, the cumulative incidence of CMV reactivation did not show any difference between groups (see Figure S1B, SDC, http://links.lww.com/TP/A951). It should be emphasized that patients in the MSC group were all

TABLE 1. Cumulative incidence of aGVHD, cGVHD, CMV reactivation, secondary neutropenia, and pulmonary infection (including etiology)

\begin{tabular}{|c|c|c|c|}
\hline & MSC group $\mathbf{n}=30$ & Controls $n=28$ & $P$ \\
\hline 1-Yr cumulative incidence of grade II-IV acute GVHD (\%) & 30 & 36 & 0.6 \\
\hline 1-Yr cumulative incidence of chronic GVHD (\%) & 67 & 52 & 0.14 \\
\hline 1-Yr cumulative incidence of CMV reactivation (\%) & 40 & 43 & 0.76 \\
\hline 1-Yr cumulative incidence of neutropenia (\%) & 14 & 4 & 0.17 \\
\hline 1-Yr cumulative incidence of pulmonary infection (\%) & 48 & 15 & 0.0074 \\
\hline Fungal $(\mathrm{n}=)$ & 6 & 1 & 0.06 \\
\hline Viral $(\mathrm{n}=)$ & 1 & 0 & 0.35 \\
\hline Bacterial $(\mathrm{n}=)$ & 3 & 2 & 0.71 \\
\hline Total $(\mathrm{n}=)$ & 19 & 6 & 0.02 \\
\hline
\end{tabular}

GVHD, graft versus host disease; CMV, cytomegalovirus. 
TABLE 2. Patient characteristics

\begin{tabular}{|c|c|c|c|}
\hline & MSC group $n=30$ & Controls $n=28$ & $P$ \\
\hline Age, yr & $54 \pm 13$ & $57 \pm 11$ & 0.40 \\
\hline BMI & $25 \pm 4$ & $26 \pm 4$ & 0.54 \\
\hline Tobacco habits (n/ex/cs) & $12 / 8 / 10$ & $8 / 13 / 7$ & 0.29 \\
\hline Comorbidities (HSCT-CI score) & $2(0-9)$ & $3(0-7)$ & 0.16 \\
\hline \multicolumn{4}{|l|}{ Underlying malignancy } \\
\hline NHL & 6 & 4 & \\
\hline CLL & 2 & 3 & \\
\hline ALL & 1 & 2 & \\
\hline CML & 0 & 1 & \\
\hline AML & 11 & 10 & \\
\hline \multicolumn{4}{|l|}{ No. cells transplanted $\left(\times 10^{6} / \mathrm{kg}\right)$} \\
\hline CD34 $4^{+}$cells & $4.3(1.0-11.7)$ & $6.1(2.6-14.5)$ & 0.05 \\
\hline $\mathrm{CD}^{+}$cells & $271(92-540)$ & $320(140-598)$ & 0.24 \\
\hline \multicolumn{4}{|l|}{ Patient/donor compatibility } \\
\hline 10/10 HLA identical (allelic level) & 0 & 28 & $<0.0001$ \\
\hline Other & 30 & 0 & \\
\hline Female donor-to-male recipient & 9 & 2 & 0.03 \\
\hline Other sex combinations & 21 & 26 & \\
\hline Aspergillosis before HSCT & 5 & 3 & 0.51 \\
\hline \multicolumn{4}{|l|}{ CMV serologic status, recipient-donor } \\
\hline
\end{tabular}

Results are expressed as mean $\pm \mathrm{SD}$ except for HCT-CI score and number of $\mathrm{CD}^{+}$and $\mathrm{CD} 34^{+}$cells transplanted expressed as median (range).

BMI, body mass index; n, non-smoker; ex, ex-smoker; cs, current smoker; HL, Hodgkin lymphoma; NHL, non-Hodgkin lymphoma; CLL, chronic lymphocytic leukemia; ALL, acute lymphoblastic leukemia; CML, chronic myeloid leukemia; AML, acute myeloid leukemia; MPD, myeloproliferative disorder; MM, multiple myeloma; PLL, prolymphocytic leukemia; HSCT, hematopoietic stem cell transplantation; CMV, cytomegalovirus.

HLA-mismatched while controls were 10/10 HLA-matched at the allelic level.

In univariate Cox analysis of the whole cohort $(n=58)$, MSC co-infusion showed a significant association with the occurrence of pulmonary infections $(\mathrm{HR}=2.96$ [1.15-7.60], $P<0.05)$ whereas aspergillosis before HSCT and female donor-to-male recipient were borderline significant $(P=0.05$ and $P=0.08$, respectively, see Table S2, SDC, http://links.lww.com/TP/A951). After multivariate analysis adjusting for aspergillosis before HSCT, female donor-to-male recipient, and MSC co-infusion, there was only a trend for an association between MSC and pulmonary infections $(P=0.09)$. The only significant risk factor was aspergillosis before HSCT (HR: 3.19 [1.08-9.43], $P<0.05$ ).

In univariate analysis, recipient $(\mathrm{HR}=7.78$ [2.31-26.3], $P=0.0009)$ and donor (HR=2.28 [1.03-5.03], $P=0.04) \mathrm{CMV}$ serostatus, but not MSC co-infusion, predicted for CMV reactivation, whereas age $(P=0.07)$ and female donor-to-male recipient $(P=0.09)$ were borderline significant. In multivariate analysis, the only parameters that appeared significant were the donor and recipient CMV positive status $(\mathrm{HR}=2.54$
[1.10-5.84], $P=0.03$ and $\mathrm{HR}=7.56$ [2.18-26.23], $P=0.001$, respectively) and female donor-to-male recipient was borderline significant (HR=2.25 [0.87-5.81], $P=0.09$ ) (see Table S2, SDC, http://links.lww.com/TP/A951).

\section{DISCUSSION}

In this study, we investigated the impact of MSC coinfusion on several clinical and laboratory outcomes of patients who underwent HSCT. To investigate the effect of MSC on the lung, we focused our analysis on pulmonary function monitoring and pulmonary infections.

At 1 year, even if all patients in the MSC group and none in the control group received a graft from HLAmismatched donors, we did not observe any difference between groups for the incidence of acute GVHD (aGVHD). Indeed, transplantation from HLA-mismatched donors is known to give less favorable outcome because of a higher incidence of GVHD. In addition, the higher proportion of the female-to-male combination, usually associated with a higher risk of chronic GVHD (cGHVD) (18), was not correlated with 
a greater incidence of cGVHD in the MSC group. These observations might attest for a protective effect of MSC against GVHD, but this remains to be studied in prospective randomized trials.

The original finding of our study is the fact that patients receiving MSC exhibited no deterioration in lung function indices over the first year of observation. There was even a slight improvement in airway flow rates and vital capacity and no change in lung diffusing capacity in the MSC group, whereas the group without MSC exhibited a significant decrease in diffusion lung capacity reaching on average $10 \%$ after 1 year. Although some animal data drew attention to the potential fibrotic effect of MSC (10), our observation attests that infused MSC did not result in excessive airway or lung remodeling that could potentially alter lung function.

By contrast, the incidence of pulmonary infections appeared to be higher in the MSC group, which was also HLAmismatched, compared to the control group that was HLA-matched. This observation could theoretically be linked to the immunosuppressive effects of MSC, GVHD, or CMV reactivation (19). However, GVHD and CMV reactivation were not predictive of lung infections, neither in univariate nor in multivariate analyses, but we have no detailed data on immune reconstitution. Likewise, neutropenia, aspergillosis before HSCT, and tobacco status, all recognized risk factors for pulmonary infections, in general (20-22) were evaluated, but only a previous episode of aspergillosis before HSCT was significantly predictive. It should be emphasized that these analyses did not investigate the impact of MSC alone but also the combined effect of HLA mismatch and MSC coinfusion, as the two factors were systematically associated. HLA mismatching could have caused a higher incidence of severe GHVD in the MSC group and hence a greater risk of fungal infections (23). However, we did not observe any difference between the groups for the incidence of aGVHD and cGVHD. Nevertheless, we cannot exclude that immune reconstitution may have been relatively impaired or delayed in the MSC group because patients were all HLA-mismatched with their donors.

Although MSC have been shown to possess antimicrobial properties, this ability has been essentially observed in vitro or in animal models and in ex vivo models of human lung tissues (24). Moreover, this property was mainly described against bacterial, viral, and parasitic infections (25-27). Interestingly, we encountered a higher incidence of fungal infections in the MSC group. Although not statistically significant, the impact of MSC could have been significant if we did not have to exclude from the study two cases with aspergillosis because they could not undergo PFT. However, patients receiving MSC were also HLA-mismatched with their donors, which could also favor infection. Forslow et al. also found an association between MSC co-infusion and pneumoniarelated death (28). The majority of patients had mold-related pneumonia, but authors were not able to prove the relation with MSC co-infusion because of low patient numbers in this study. However, in a further paper, the use of MSC was associated with a higher incidence of invasive fungal infections in case of severe aGVHD (29), which is in line with our results.

Contrary to pulmonary infections, MSC co-infusion did not have an impact on CMV reactivation, and this was attested by the fact that the cumulative incidence of CMV reactivation did not differ from the control group. A similar finding was observed in a recent paper by Lucchini et al. (30), which demonstrated that MSC did not interfere with antiviral responses in vivo. The only parameters found to be strongly predictive of CMV reactivation in this study were donor and recipient CMV positive status, which are widely recognized as risk factors for such reactivation (31).

In conclusion, the main finding of our study is the fact that patients who underwent HSCT with MSC co-infusion after NMA showed no deterioration of lung function over a period of 1 year after transplantation. Nevertheless, longer follow-up in larger groups of patients would be required to formally exclude any lung toxicity of MSC, in particular in relation with cGHVD. However, the pulmonary infection rate (mainly the occurrence of fungal infections) appeared to be increased. This indicates the need for prolonged antifungal prophylaxis and close monitoring of pulmonary infections in patients after HSCT and MSC cotransplantation.

\section{MATERIALS AND METHODS}

\section{Subjects}

Patients who underwent HSCT after NMA conditioning in the University Hospital Center of Liege between December 2006 and December 2012 were screened for the following inclusion criteria: (1) conditioning with total body irradiation (TBI) 2 Gy and fludarabine, (2) immunosuppression with mycophenolate mofetil (MMF) and tacrolimus, (3) transplantation with peripheral blood stem cells (PBSC), (4) unrelated donor, and (5) minimal follow-up of 100 days. Thirty-eight patients met the inclusion criteria in the MSC group. Among them, eight were excluded because they did not have lung function assessment 3 months after HSCT. Indeed, three patients died before day 100 (one died of graft rejection and sepsis, one of relapse, and one of GVHD and sepsis associated with organ failure). The other five patients were not able to perform lung function tests at day 100 (two had invasive lung aspergillosis, complicated by renal failure in one; two had a relapse associated with severe encephalopathy in one; one had severe GVHD and organ failure). Consequently, 30 patients remained eligible in the MSC group. They took all part in one of two consecutive clinical trials investigating the safety and efficacy of MSC co-infusion at time of HSCT from mismatched unrelated donors after NMA conditioning (1). In the non-MSC group, 35 patients also undergoing HSCT after NMA conditioning were included. However, three died before 100 days (two of relapse, one of GVHD associated with sepsis and organ failure), and four did not perform lung function tests because of poor health status (two had a relapse combined with sepsis, one had severe GVHD associated with organ failure, and one had GVHD combined with encephalopathy). Therefore, 28 patients remained eligible in the control group. Characteristics of the patients are presented in Table 2. All patients included in the NMA MSC trial had HLA mismatches (one allele up to two antigens), whereas all controls were 10/10 HLA-matched at allelic level.

MSC were cultured as described previously $(1,32)$. The conditioning regimen consisted of fludarabine $90 \mathrm{mg} / \mathrm{m}^{2}$, followed by a single dose of 2 Gy TBI administered on day 0 before infusion of cells. MSC were infused first, followed by PBSC infused at least 60 to $120 \mathrm{~min}$ later. MMF was administered orally from day 0 through day 42 at the dose of $15 \mathrm{mg} / \mathrm{kg}$ three times a day. Tacrolimus was given orally at the dose of $0.06 \mathrm{mg} / \mathrm{kg}$ twice a day starting on day -3 until day 180 and then progressively tapered to be definitely discontinued by day 365 in the absence of GVHD. The conditioning regimen and postgrafting immunosuppression used were identical in the MSC and the control groups.

The diagnosis and clinical grading of aGVHD were performed according to standard criteria $(33,34)$. Diagnosis and grading of cGVHD were made using the National Institute of Health consensus criteria (35).

All subjects gave written informed consent for participation as well as for collection and analyses of posttransplant data. The MSC clinical trials were approved by the Ethics Committee of the University of Liege. 


\section{Pulmonary Function Assessment}

At each time point, subjects underwent a global lung function assessment using a body box plethysmography (Sensormedics, Vmax series 22; Viasyhealthcare, Yorba Linda, CA) allowing to measure flow rates, lung volumes, and diffusion capacity according to ATS/ERS standard criteria (36-38). Spirometry (measure of forced expiratory volume in 1 sec: $\mathrm{FEV}_{1}$ and forced vital capacity) was performed before and after $400 \mu \mathrm{g}$ inhaled salbutamol metered dose inhaler administered through a Volumatic. Diffusion for carbon monoxide (DLCO) was measured by the single breath washout technique and corrected for the hemoglobin content. FeNO was measured using a chemoluminescence analyzer (NIOX; Aerocrine, Stockholm, Sweden) at a flow rate of $50 \mathrm{~mL} / \mathrm{sec}$, in accordance with the recommendation of the ATS/ERS task force (39).

\section{Pulmonary Infections and CMV Reactivation}

Standard prophylaxis against infections was used (40), and disease evaluation was routinely carried out on days $40,100,180$, and 365 . Pulmonary infections were diagnosed based on respiratory symptoms, microbial analysis of bronchoalveolar lavage, and chest radiography or CT scan, or both. Bronchitis leading to hospitalization and pneumonia were recorded. The day of the first CMV reactivation episode, defined as the first viral load greater than 1,000 copies/ $\mathrm{mL}$ by PCR, was also recorded and positive patients were treated preemptively with ganciclovir. Secondary neutropenia was defined as an episode of at least 2 weeks with absolute neutrophil count less than 500 cells $/ \mu \mathrm{L}$ occurring at least 1 month after HSCT.

\section{Statistical Analysis}

Categorical parameters were compared using the chi-square test. Comparisons between PFT values before and after HSCT were performed using paired $t$ tests or Wilcoxon matched-pair signed rank tests for the FeNO values. Intergroup comparisons were made using unpaired $t$ tests or MannWhitney tests as appropriate. Cumulative incidences of aGVHD, cGHVD, neutropenia, CMV reactivation, and pulmonary infection were calculated as previously described (41), taking death as competing event. The impact of MSC co-infusion (and correlatively of HLA mismatch because of the linkage between the two characteristics) on CMV reactivation and pulmonary infections was assessed in univariate and multivariate Cox models. Factors analyzed in univariate analysis were as follows: patient age, gender, tobacco habits, acute leukemia versus other diagnoses, number of CD34+ cells transplanted, aspergillosis before HSCT, secondary neutropenia, grade II-IV aGVHD, female donor-to-male recipient versus other sex combinations, and MSC co-infusion. CMV reactivation was also assessed as risk factor for pulmonary infection, and pulmonary infection and donor and recipient CMV serostatus were evaluated as risk factors for CMV reactivation. Factors with a $P$ value less than 0.10 were then introduced into multivariate analysis. Differences were considered statistically significant when a two-sided $P$ value was less than 0.05 . Statistical analyses were carried out with GraphPad Prism (GraphPad Software, San Diego, CA) and SAS version 9.3 (SAS Institute, Cary, NC).

\section{ACIKNOWLEDGIMENTS}

The authors would like to thank all participants of the study and the lung function laboratory of the University Hospital Center of Liege for their help in the collection of the data.

\section{REFERENCES}

1. Baron F, Lechanteur C, Willems E, et al. Cotransplantation of mesenchymal stem cells might prevent death from graft-versus-host disease (GVHD) without abrogating graft-versus-tumor effects after HLA-mismatched allogeneic transplantation following nonmyeloablative conditioning. Biol Blood Marrow Transplant 2010; 16: 838.

2. von Bahr L, Sundberg B, Lonnies L, et al. Long-term complications, immunologic effects, and role of passage for outcome in mesenchymal stromal cell therapy. Biol Blood Marrow Transplant 2012; 18: 557.

3. Bernardo ME, Cometa AM, Locatelli F. Mesenchymal stromal cells: a novel and effective strategy for facilitating engraftment and accelerating hematopoietic recovery after transplantation? Bone Marrow Transplant 2012; 47: 323.
4. Lin Y, Hogan WJ. Clinical application of mesenchymal stem cells in the treatment and prevention of graft-versus-host disease. Adv Hematol 2011; 2011: 427863.

5. Sundin M, Barrett AJ, Ringden O, et al. HSCT recipients have specific tolerance to MSC but not to the MSC donor. J Immunother 2009; 32: 755.

6. Schrepfer S, Deuse T, Reichenspurner H, et al. Stem cell transplantation: the lung barrier. Transplant Proc 2007; 39: 573.

7. Aguilar S, Nye E, Chan J, et al. Murine but not human mesenchymal stem cells generate osteosarcoma-like lesions in the lung. Stem Cells 2007; 25: 1586.

8. Djouad F, Plence P, Bony C, et al. Immunosuppressive effect of mesenchymal stem cells favors tumor growth in allogeneic animals. Blood 2003; 102: 3837.

9. Yan X, Liu Y, Han Q, et al. Injured microenvironment directly guides the differentiation of engrafted Flk-1(+) mesenchymal stem cell in lung. Exp Hematol 2007; 35: 1466.

10. Salazar KD, Lankford SM, Brody AR. Mesenchymal stem cells produce Wnt isoforms and TGF-betal that mediate proliferation and procollagen expression by lung fibroblasts. Am J Physiol Lung Cell Mol Physiol 2009; 297: L1002.

11. Gupta N, Su X, Popov B, et al. Intrapulmonary delivery of bone marrow-derived mesenchymal stem cells improves survival and attenuates endotoxin-induced acute lung injury in mice. J Immunol 2007; 179: 1855.

12. Rojas $\mathrm{M}, \mathrm{Xu}$ J, Woods $\mathrm{CR}$, et al. Bone marrow-derived mesenchymal stem cells in repair of the injured lung. Am J Respir Cell Mol Biol 2005; 33: 145 .

13. Jungebluth P, Luedde M, Ferrer E, et al. Mesenchymal stem cells restore lung function by recruiting resident and nonresident proteins. Cell Transplant 2011; 20: 1561.

14. Iyer SS, Co C, Rojas M. Mesenchymal stem cells and inflammatory lung diseases. Panminerva Med 2009; 51: 5.

15. Chien JW, Madtes DK, Clark JG. Pulmonary function testing prior to hematopoietic stem cell transplantation. Bone Marrow Transplant 2005; 35: 429.

16. Beeh KM, Kornmann O, Lill J, et al. Induced sputum cell profiles in lung transplant recipients with or without chronic rejection: correlation with lung function. Thorax 2001; 56: 557.

17. Travers J, Marsh S, Aldington S, et al. Reference ranges for exhaled nitric oxide derived from a random community survey of adults. Am J Respir Crit Care Med 2007; 176: 238.

18. Flowers ME, Inamoto Y, Carpenter PA, et al. Comparative analysis of risk factors for acute graft-versus-host disease and for chronic graftversus-host disease according to National Institutes of Health consensus criteria. Blood 2011; 117: 3214.

19. Nichols WG, Corey L, Gooley T, et al. High risk of death due to bacterial and fungal infection among cytomegalovirus (CMV)-seronegative recipients of stem cell transplants from seropositive donors: evidence for indirect effects of primary CMV infection. J Infect Dis 2002; 185: 273.

20. Fukuda T, Boeckh M, Guthrie KA, et al. Invasive aspergillosis before allogeneic hematopoietic stem cell transplantation: 10-year experience at a single transplant center. Biol Blood Marrow Transplant 2004; 10: 494.

21. Bagaitkar J, Demuth DR, Scott DA. Tobacco use increases susceptibility to bacterial infection. Tob Induc Dis 2008; 4: 12.

22. Donowitz GR, Maki DG, Crnich CJ, et al. Infections in the neutropenic patient-new views of an old problem. Hematology Am Soc Hematol Educ Program 2001; 39: 113.

23. Blennow O, Remberger M, Klingspor L, et al. Randomized PCR-based therapy and risk factors for invasive fungal infection following reduced-intensity conditioning and hematopoietic SCT. Bone Marrow Transplant 2010; 45: 1710.

24. Lee JW, Krasnodembskaya A, McKenna DH, et al. Therapeutic effects of human mesenchymal stem cells in ex vivo human lungs injured with live bacteria. Am J Respir Crit Care Med 2013; 187: 751.

25. Meisel R, Brockers S, Heseler K, et al. Human but not murine multipotent mesenchymal stromal cells exhibit broad-spectrum antimicrobial effector function mediated by indoleamine 2,3-dioxygenase. Leukemia $2011 ; 25: 648$.

26. Krasnodembskaya A, Song Y, Fang X, et al. Antibacterial effect of human mesenchymal stem cells is mediated in part from secretion of the antimicrobial peptide LL-37. Stem Cells 2010; 28: 2229.

27. Auletta JJ, Deans RJ, Bartholomew AM. Emerging roles for multipotent, bone marrow-derived stromal cells in host defense. Blood 2012; 119: 1801. 
28. Forslow U, Blennow O, LeBlanc K, et al. Treatment with mesenchymal stromal cells is a risk factor for pneumonia-related death after allogeneic hematopoietic stem cell transplantation. Eur J Haematol 2012; 89: 220.

29. Remberger M, Ringden O. Treatment of severe acute graft-versus-host disease with mesenchymal stromal cells: a comparison with non-MSC treated patients. Int J Hematol 2012; 96: 822.

30. Lucchini G, Dander E, Pavan F, et al. Mesenchymal stromal cells do not increase the risk of viral reactivation nor the severity of viral events in recipients of allogeneic stem cell transplantation. Stem Cells Int 2012; 2012: 690236 .

31. George B, Pati N, Gilroy N, et al. Pre-transplant cytomegalovirus (CMV) serostatus remains the most important determinant of CMV reactivation after allogeneic hematopoietic stem cell transplantation in the era of surveillance and preemptive therapy. Transpl Infect Dis 2010; 12: 322.

32. Le Blanc K, Frassoni F, Ball L, et al. Mesenchymal stem cells for treatment of steroid-resistant, severe, acute graft-versus-host disease: a phase II study. Lancet 2008; 371: 1579.

33. Baron F, Maris MB, Sandmaier BM, et al. Graft-versus-tumor effects after allogeneic hematopoietic cell transplantation with nonmyeloablative conditioning. J Clin Oncol 2005; 23: 1993.

34. Mielcarek M, Martin PJ, Leisenring W, et al. Graft-versus-host disease after nonmyeloablative versus conventional hematopoietic stem cell transplantation. Blood 2003; 102: 756.
35. Filipovich AH, Weisdorf D, Pavletic S, et al. National Institutes of Health consensus development project on criteria for clinical trials in chronic graft-versus-host disease: I. Diagnosis and staging working group report. Biol Blood Marrow Transplant 2005; 11: 945.

36. Macintyre N, Crapo RO, Viegi G, et al. Standardisation of the singlebreath determination of carbon monoxide uptake in the lung. Eur Respir J 2005; 26: 720.

37. Miller MR, Crapo R, Hankinson J, et al. General considerations for lung function testing. Eur Respir J 2005; 26: 153.

38. Wanger J, Clausen JL, Coates A, et al. Standardisation of the measurement of lung volumes. Eur Respir J 2005; 26: 511.

39. ATS/ERS recommendations for standardized procedures for the online and offline measurement of exhaled lower respiratory nitric oxide and nasal nitric oxide, 2005. Am J Respir Crit Care Med 2005; 171: 912.

40. Castermans E, Baron F, Willems E, et al. Evidence for neo-generation of $\mathrm{T}$ cells by the thymus after non-myeloablative conditioning. Haematologica 2008; 93: 240.

41. Gooley TA, Leisenring W, Crowley J, et al. Estimation of failure probabilities in the presence of competing risks: new representations of old estimators. Stat Med 1999; 18: 695.

42. Kahl C, Storer BE, Sandmaier BM, et al. Relapse risk in patients with malignant diseases given allogeneic hematopoietic cell transplantation after nonmyeloablative conditioning. Blood 2007; 110: 2744.

\title{
Instructions for Authors-Key Guidelines
}

\author{
Financial Disclosure and Products Page
}

Authors must disclose any financial relationship with any entity or product described in the manuscript (including grant support, employment, honoraria, gifts, fees, etc.) Manuscripts are subject to peer review and revision may be required as a condition of acceptance. These instructions apply to all submissions.

\section{Manuscript Review and Publication}

Using an electronic on-line submission and peer review tracking system, Transplantation is committed to rapid review and publication. The average time to first decision is now less than 21 days. Time to publication of accepted manuscripts continues to be shortened, with the Editorial team committed to a goal of 3 months from acceptance to publication.

Submit your manuscript to Transplantation ${ }^{\circledR}$ today. The Manuscript Central submission website (http://mc.manuscriptcentral.com/transplant) helps make the submission process easier, more efficient, and less expensive for authors, and makes the review process quicker, more accessible, and less expensive for reviewers. 